\title{
Variant lambda of the severe acute respiratory syndrome coronavirus 2: A serious threat or the beginning of further dangerous mutations
}

\author{
Lukasz Szarpak ${ }^{1,2,3}$ (อ, Ivan Savytskyi ${ }^{4}$, Michal Pruc ${ }^{3,5}$, Anatolyi Gozhenko ${ }^{4}$,

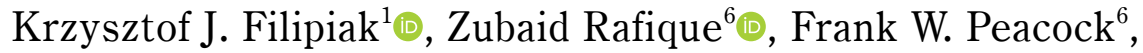 \\ Olayinka S. Ilesanmi $^{7,8}{ }^{(1)}$, Francesco Chirico $^{9,10}{ }^{(0)}$ \\ ${ }^{1}$ Institute of Outcomes Research, Maria Sklodowska-Curie Medical Academy, Warsaw, Poland \\ ${ }^{2}$ Research Unit, Maria Sklodowska-Curie Bialystok Oncology Center, Bialystok, Poland \\ ${ }^{3}$ Polish Society of Disaster Medicine, Warsaw, Poland \\ ${ }^{4}$ International European University, Kiev, Ukraine \\ ${ }^{5}$ Institute of Outcomes Research, Polonia University, Warsaw, Poland
}

${ }^{6}$ Henry JN Taub Department of Emergency Medicine, Baylor College of Medicine, Houston, TX, United States

${ }^{7}$ Department of Community Medicine, College of Medicine, University of Ibadan, Oyo State, Nigeria

${ }^{8}$ Department of Community Medicine, University College Hospital, Ibadan, Oyo State, Nigeria

${ }^{9}$ Post-graduate School of Occupational Health, Università Cattolica del Sacro Cuore, Rome, Italy

${ }^{10}$ Health Service Department, Italian State Police, Milan, Italy

In mid-June, the World Health Organization (WHO) recognized the Lambda variant as a socalled Variants of Interest (VOI) [1]. In addition to Lambda, this list includes six others: Epsilon (B.1.427/B.1.429, formerly Californian), Iota (B.1.526, New York), and Theta (P.3, Filipino). This means that these variants have mutations that affect, inter alia, the ease of transmission of the pathogen, the severity of the disease, the ability to avoid vaccines, or misleading diagnostic tests. On the basis of genome sequencing, it was estimated that this variant contains 27 mutations ( 1 in ORF1a - deletion 3675-3677; 7 in the gene encoding protein S - deletion 246-252, G75V, T76I, L452Q, F490S, D614G and T859N as well as 19 other mutations which are observed in various known variants of the SARS-2 coronavirus). Among the Lambda variant mutations, the L452Q mutation was identified, which is similar to the L452R mutation observed in the Delta and Epsilon variants [2]. A new variant of interest in severe acute respiratory syndrome coronavirus 2 (SARS--CoV-2), designated Lambda, has spread in recent months in some South American countries, but its virological and evolutionary features remain unknown. The Lambda variant spike protein may increase viral infectivity, which is attributed to the mutations designated T76I and L452Q. The RSYLTPGD246$253 \mathrm{~N}$ mutation, which is a unique 7 -amino acid deletion in the $\mathrm{N}$-terminal domain of the Lambda variant spike protein, avoids neutralizing antibodies. As the SARS-CoV-2 Lambda variant has spread dominantly in line with the increasing frequency of isolates carrying the RSYLTPGD246-253N mutation, these data suggest that the insertion of the RSYLTPGD246-253N mutation is closely related to the massive spread of Lambda variant infection in South America. Thus, the spike protein of the Lambda variant increases its infectivity, and the T76I and L452Q mutations are responsible for this property, and together with the RSYLTPGD246$253 \mathrm{~N}$ and F490S mutation they confer resistance to antiviral antibodies [3]. The effect of such mutations on infectivity and immune escape from

Address for correspondence: Lukasz Szarpak, Assoc. Prof., PhD, DPH, MBA, DBA, LLM, Institute of Outcomes Research, Maria Sklodowska-Curie Medical Academy, ul. Solidarności 12, 03-411 Warszawa, Poland, e-mail: lukasz.szarpak@gmail.com 
neutralizing antibodies is completely unknown to us. The studies showed an increased infectivity of the Lambda variant, which was even higher than that of D614G (a line of Alpha and Gamma variants). Compared to the wild type (line A), the neutralization was reduced 3.05-fold for the Lambda variant, while it was 2.33 -fold for the Gamma variant and 2.03-fold for the Alpha variant. These results indicate that mutations present in the spike protein of the Lambda variant of interest result in increased infectivity and immune escape from neutralizing antibodies induced by an inactivated CoronaVac vaccine (not available in the European Union or the United States). These results show that in countries with high levels of SARS-CoV-2 infections, genetic surveillance should be associated with the identification of new isolates harboring mutations in the spike protein gene and immunological studies to determine the effect of these mutations on immune escape and breaking vaccine immunity [4]. Fortunately, the Lambda variant remains susceptible to neutralization by vaccine-induced mRNA antibodies. Lambda variant was more infectious and was neutralized by convalescent sera and vaccine-induced antibodies with a relatively small $2.3-3.3$-fold decrease in titer, however this decrease was present. therefore, vaccination based on mRNA technology should be used universally and the whole world should focus on their universal use as well as on enabling their use in poorer countries [5]. According to Johns Hopkins Coronavirus Resource Center, Peru currently has the highest mortality rate, which is 600 deaths per 100,000 infected with COVID-19, in addition, the WHO reports that in Peru, the Lambda varianthas affected about $81 \%$ of patients since April, which additionally raises concern about the spread of this variant around the world [6].

We must pay special attention to the Lambda variant of the SARS-CoV-2 due to its possible es- cape from the surveillance of the host's immune response and possible ineffectiveness or limited effectiveness of preventive vaccinations. We should also consider a possible change in vaccination in countries such as Peru and the use of the most effective of them, such as those produced by Pfizer and Moderna, based on mRNA technology. Vaccinating the society as quickly as possible and with the most effective preparations should reduce the transmission of the virus and the risk of mutations that will no longer be resistant to the preventive measures we currently know. In the case of the Lambda variant, it is also necessary to consider the introduction of compulsory wearing of masks and travel restrictions, as well as implementing quarantine for returnees, regardless of vaccination status.

\section{Conflict of interest: None declared}

\section{References}

1. "Tracking SARS-CoV-2 variants". who.int. World Health Organization. Updated frequently. https://www.who.int/en/activities/ tracking-SARS-CoV-2-variants/ (Access: 2 August 2021).

2. Wink PL, Volpato FC, Monteiro FL, et al. First identification of SARS-CoV-2 Lambda (C.37) variant in Southern Brazil. Infect Control Hosp Epidemiol. 2021 [Epub ahead of print]: 1-7, doi: 10.1017/ice.2021.390, indexed in Pubmed: 34470685.

3. Kimura I, Kosugi $\mathrm{Y}, \mathrm{Wu}$ J, et al. SARS-CoV-2 Lambda variant exhibits higher infectivity and immune resistance. bioRxiv. 2021; 2021.07.28.454085, doi: 10.1101/2021.07.28.454085.

4. Acevedo M, Alonso-Palomares L, Bustamante A, et al. Infectivity and immune escape of the new SARS-CoV-2 variant of interest Lambda. medRxiv. 2021; 2021.06.28.21259673, doi: 10.1101/2021.06.28.21259673.

5. Tada T, Zhou H, Dcosta B, et al. SARS-CoV-2 Lambda Variant Remains Susceptible to Neutralization by mRNA Vaccineelicited Antibodies and Convalescent Serum. bioRxiv. 2021; 2021.07.02.450959, doi: 10.1101/2021.07.02.450959.

6. Weekly epidemiological update on COVID-19 - 15 June 2021 Edition 4415 June 2021 | Emergency Situational Update. https:// www.who.int/publications/m/item/weekly-epidemiological-update-on-covid-19---15-june-2021 (Access: 2 August 2021). 